TIPA. Travaux interdisciplinaires sur la parole et le langage

$35 \mid 2019$

Emo-langages

\title{
La notion de pertinence au défi des effets émotionnels
}

Relevance and emotional effects

Louis de Saussure et Tim Wharton

\section{OpenEdition}

1 Journals

Édition électronique

URL : http://journals.openedition.org/tipa/3068

DOI : 10.4000/tipa.3068

ISSN : 2264-7082

Éditeur

Laboratoire Parole et Langage

Référence électronique

Louis de Saussure et Tim Wharton, "La notion de pertinence au défi des effets émotionnels », TIPA. Travaux interdisciplinaires sur la parole et le langage [En ligne], 35 | 2019, mis en ligne le 15 juillet 2019, consulté le 26 février 2020. URL : http://journals.openedition.org/tipa/3068 ; DOI : 10.4000/tipa.3068

Ce document a été généré automatiquement le 26 février 2020.

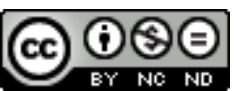

La revue TIPA. Travaux interdisciplinaires sur la parole et le langage est mise à disposition selon les termes de la licence Creative Commons Attribution - Pas d'Utilisation Commerciale - Pas de Modification 4.0 International. 


\title{
La notion de pertinence au défi des effets émotionnels
}

\author{
Relevance and emotional effects \\ Louis de Saussure et Tim Wharton
}

\section{Introduction}

1 Les théories pragmatiques contemporaines, d'inspiration gricéenne, ont pour objectif de rendre compte des processus interprétatifs en jeu dans la communication. La Théorie de la pertinence de Sperber \& Wilson (1986/1995), qui nous intéressera tout particulièrement, cherche aujourd'hui à aborder un champ qui nous semble crucial : les aspects «non propositionnels » du sens. Or il nous semble important de souligner que ce qui relève de la dimension affective de la communication, et notamment le partage des émotions, relève par excellence du sens non propositionnel.

2 La question posée dans cet article est la suivante : comment une théorie pragmatique d'inspiration cognitive comme la Théorie de la pertinence pourrait-elle rendre compte des émotions véhiculées par la communication?

3 Pour l'instant, nous nous en tiendrons provisoirement à une notion intuitive d'émotion, entendue comme un état mental affectif, une forme particulière d'attitude ressentie. Nous détaillerons dans la section 6 ce qu'il convient précisément d'entendre par ce terme d'émotion. Précisons d'emblée que nous nous intéressons non pas à la description des émotions (du type de "Je suis furieux contre toi») mais plutôt aux moyens linguistiques d'expression directe de ces émotions (du type de « Enfoiré ! »).

4 En linguistique, une immense littérature existe au sujet de ces effets, parfois regroupés sous la notion d'expressivité, mais celle qui provient de l'héritage gricéen, et de manière plus générale, des approches analytiques de la sémantique et de la pragmatique, est remarquablement pauvre. Font exception un certain nombre de travaux qui décrivent le comportement linguistique particulier des « expressifs » (par exemple Potts, 2007) et quelques articles qui ouvrent des pistes pour envisager la question du non- 
propositionnel. Cette question est notamment récurrente au sein de la Théorie de la pertinence et se lie au problème, immédiatement connexe, des expressions "descriptivement ineffables ", c'est-à-dire intraduisibles en contenus propositionnels (Blakemore, 2011; Wharton, 2015; de Saussure \& Schulz, 2009), des «impressions » (Sperber \& Wilson, 2015), essentiellement conçues comme liées à des effets de saillance ou de manifesteté ( $c f$. infra), ou encore à celle des effets littéraires (Cave \& Wilson, 2018). C'est à explorer ces pistes et proposer des solutions nouvelles que cet article est consacré, afin d'intégrer dans ce panorama des effets communicatifs proprement « émotionnels».

5 Dans un premier temps, nous exposons et discutons les acquis principaux de la littérature sur les effets non-propositionnels, affectifs, émotionnels, qui peuvent, et doivent, être intégrés dans une approche pragmatique cognitive contemporaine. Ensuite, nous nous penchons sur un cas d'espèce où une grossière erreur pragmatique a conduit un auditoire vers des émotions non souhaitées par le locuteur (section 3) avant de discuter de la question de la dimension proprement expressive du langage (section 4). Dans la section 5, nous rappelons les principes de la Théorie de la pertinence de Sperber \& Wilson (1986/1995), afin de montrer que dans sa configuration actuelle, elle n'est pas en mesure de traiter de manière satisfaisante les aspects affectifs de la communication. La section 6 est consacrée à détailler ce qu'il faut entendre par émotions et effets émotionnels. Dans la section 7, nous évoquons les effets émotionnels comme moyens de dépasser les limites du sens propositionnel, et tentons d'établir un lien avec certains types de discours figuré, ce qui nous permet de revenir sur le cas du malentendu évoqué auparavant car il fait intervenir du style indirect libre mal maîtrisé. La section 8 est consacrée à la notion de partage des émotions et à une hypothèse sur la manière dont elles sont activées par les expressifs via une forme de simulation, ce que nous tentons d'intégrer à la théorie de la pertinence en proposant une solution pour les effets émotionnels relativement au principe de pertinence. Nous suggérons qu'ils activent une validation expérientielle et non pas cognitiveconceptuelle. Nous y évoquons également l'interaction entre effets cognitifs et effets émotionnels.

\section{Au-delà du sens propositionnel}

6 Si les humains ont une propension remarquable à communiquer entre eux, et que cette propension participe de la complexité des sociétés humaines, cela tient notamment à la complexité des outils de communication, au premier plan desquels le langage, qui par sa puissance à la fois structurelle et évocatrice, conduit à des effets de représentation très sophistiqués d'intentions et de pensées à destination d'autrui. Cependant, le domaine d'étude de la communication verbale recouvre en principe une variété de faits passablement hétérogènes: communication d'opinions, d'actions (l'engagement par une promesse, par exemple), d'arguments, d'états de choses réels ou fictifs, d'états affectifs et émotionnels, d'attitudes, d'appréciations, de modalités diverses, etc., de sorte qu'il est difficile d'admettre a priori que les méthodologies d'investigation développées par les chercheurs puissent s'appliquer indifféremment à eux tous.

7 Malgré le fait que la communication d'informations au sujet d'états émotionnels joue de manière évidente un rôle central dans l'interaction, les approches linguistiques contemporaines qui traitent spécifiquement de la communication langagière (le 
langage en usage) ne lui accordent pas la place qu'elle mérite. Cela tient au fait que leurs outils, et leur philosophie générale, ne concernent que la partie informationnelle du sens. Or il est évident qu'il y a une différence cruciale entre un énoncé comme (1) ou (3), qui décrivent un état mental avec des propositions, et (2) ou (4), qui les expriment de manière directe (ils ne sont d'ailleurs pas vraiment équivalents) :

(1) Je suis furieux contre toi.

(2) Enfoiré !

(3) J'ai mal.

(4) Aïe !

8 Les contributions des approches dominantes en sémantique et en pragmatique de tradition formelle ou cognitive à la question des états mentaux non propositionnels (représentés par exemple dans des énoncés comme (2) ou (4)), sont essentiellement descriptives ; mais dans la ligne de la philosophie formelle depuis Frege, ces questions sont tout simplement jugées soit inexplicables soit futiles. Ce n'est pas très surprenant : tant la sémantique formelle, "vériconditionnelle "1 que la pragmatique d'inspiration gricéenne sont essentiellement propositionnalistes, c'est-à-dire que leur champ d'investigation se circonscrit en quelque sorte de lui-même à l'intérieur du monde de la proposition. Cela tient au fait que seules les propositions semblent permettre des computations et des analyses, d'une part, et qu'elles sont le composant par excellence des pensées auxquelles il est possible de faire correspondre des phrases.

9 Ce postulat courant s'étend vers la notion de communication, et est assez bien résumé par Stephen Levinson:

La portée complète d'une théorie de la communication correspond au sens nonnaturel de Grice (1957). (...) Le sens non-naturel, ou quoi que ce soit du même genre, délimite le territoire des effets communicatifs qui est du ressort d'une théorie de la communication. (Levinson, $2000: 12-13$, notre trad.).

10 De la sorte, une théorie de la communication ne devrait porter que sur l'information au sens propositionnel, qui surgit de conventions sémantiques et de raisonnements pragmatiques explicitement reconstructibles.

11 Pourtant, l'espace du sens non propositionnel est reconnu par une littérature abondante et ancienne qui le signale comme au moins aussi important sur le plan anthropologique que le sens propositionnel avec lequel il s'articule. Les traditions francophones en particulier, issues du structuralisme saussurien plutôt que du formalisme analytique frégéen, reconnaissent en Charles Bally le père de la linguistique de l'affectivité, par les observations que son œuvre développe et qui forme les fondements d'une philosophie de la communication langagière affective (Bally, 1905 ; 1910 ; 1923). Notamment, Bally fait une distinction entre le contenu propositionnel qu'il appelle le dictum et la manière de dire, qui contient les éléments expressifs, qu'il appelle le modus. D'autres linguistes de cette époque ont, comme lui, critiqué l'« orientation idéationnelle» de la sémantique qui dominait déjà, suggérant que 
l'étude de la dimension expressive était au moins aussi importante que celle de la dimension cognitive-propositionnelle (Erdman, 1900 ; van Ginneken, 1907). Il n'est pas raisonnable d'imaginer qu'une théorie de la communication doive laisser non étudié ce qui fait l'un des aspects les plus essentiels de l'interaction humaine. Nous prenons donc le contrepied de l'affirmation défaitiste de Levinson.

12 Mais comment articuler des intuitions du type de celles de Bally, datant du début du $\mathrm{XX}^{\mathrm{e}}$ siècle, avec les avancées de la pragmatique d'aujourd'hui, inspirée par les travaux de Paul Grice dans les années cinquante et soixante, et qui forme, sous diverses variantes, le fonds commun de la recherche sur le sens en se liant étroitement à la dimension neurocognitive de l'interprétation? La même question se pose pour la constellation d'autres travaux qui se situent dans la même lignée et ont considéré avec attention différents éléments de sens qui ne relèvent pas du propositionnel, comme la théorie de l'énonciation de Benveniste (1970). Ducrot (cf. Ducrot, 1972; 1984) est un autre exemple du mariage qui s'opère entre les aspects comportementaux du langage, qui peuvent faire intervenir des éléments qui échappent à l'analyse propositionnelle, par exemple les effets d'identification avec des énonciateurs fictifs dans le teatrum mundi de l'interaction, et la communication d'informations propositionnelles. Cependant, articuler une idée de sens lié à l'émotion avec une théorie pragmatique comme la Théorie de la pertinence de Sperber \& Wilson (1986/95), qui nous servira ici de point d'ancrage, demande de la mettre face au défi de passer la barrière de l'effet " cognitif » pour envisager le territoire du conatif, de l'affectif. Cet objectif est jusqu'ici à peine ébauché (Moeschler, 2009) mais quelques propositions plus récentes émergent (Wharton, 2009; 2015; Wharton \& Strey, 2017; Sperber \& Wilson, 2015; Briens \& de Saussure, 2018).

13 L'importance de cette dimension émotionnelle n'est évidemment pas à justifier, mais qu'on en juge par cette remarque de Robert Badinter à propos de l'argumentation de l'avocat en cour d'assises :

Si par malheur vous lisez, vous êtes perdu. (...). Si j'ai pu réussir à les sauver, tous, [six hommes risquant la peine de mort], ce n'est pas parce que la forme était parfaite, ou l'argumentation, c'est pour une autre raison. C'est que vous vous adressez à eux, comme disent les Italiens, "le visage nu ». Ils doivent sentir que c'est un homme qui parle à des femmes et des hommes [le jury], que c'est un homme qui les met en garde contre le piège qui leur est tendu dans lequel ils vont s'engouffrer. Que c'est un homme qui leur dit: « Ne faites pas ça ».

(Robert Badinter, sur le plateau de La Grande Librairie, France 2 Télévision, octobre 2018. Soulignements d'emphase verbale).

14 Le vaste champ de recherche constitué par les heuristiques cognitives depuis les travaux fondateurs de psychologues comme Tversky \& Kahneman (1974) ou Petty \& Cacciopo (1986) se lie aujourd'hui de plus en plus clairement avec la question des processus interprétatifs (de Saussure, 2005a ; Oswald \& Maillat, 2009; Sperber et al., 2010) et interroge de plus en plus souvent le rôle des émotions dans la communication (Dezecache et al., 2013, inter alia) ainsi que dans l'argumentation et la persuasion (Plantin, 2011 ; Micheli, 2010). Mais le lien entre la partie propositionnelle et la partie non-propositionnelle du sens et leur rôle respectif dans la communication reste à élucider.

15 Nous proposons de partir d'une étude de cas afin d'aborder la notion d'effets émotionnels. 


\section{Le cas Jenninger}

16 Le 10 novembre 1988, Philipp Jenninger, alors Président du parlement ouest-allemand à Bonn, donne un discours officiel à l'occasion du cinquantième anniversaire de la Nuit de Cristal. Son discours commence par une évocation des raisons pour lesquelles, selon lui, le National-Socialisme a pu séduire l'imaginaire de nombreux Allemands à l'époque. Le style rhétorique choisi avait pour but, manifestement, de représenter d'une manière aussi fidèle que possible la perspective d'un Allemand « ordinaire » des années trente qui serait favorable au mouvement Nazi. Son intention, bien sûr, était de se dissocier du point de vue ainsi représenté.

17 Mimant le ton et l'attitude supposés de tels partisans, Jenninger évoque ce qui pouvait leur sembler si «fascinant» (dans ses termes) dans le nazisme. Utilisant la forme du style indirect libre, qui consiste à rendre la perspective interne du locuteur cité sans marque explicite de discours ou pensée rapporté (comme les guillemets du discours direct ou le verbe de parole ou de pensée du discours indirect), il dit :

Partant du chômage de masse est venu le plein-emploi, de la misère de masse, quelque chose comme la prospérité pour la partie la plus large de la population. $\mathrm{Au}$ lieu du malheur et du désespoir, c'est l'optimisme et la confiance qui règnent. Hitler n'a-t-il pas réalisé ce que Guillaume II n'avait fait qu'annoncer, à savoir conduire les Allemands vers des temps glorieux? N'était-il pas vraiment l'élu de la Providence, un chef tel que la Providence n'en donne aux peuples qu'une fois tous les mille ans $?^{2}$

18 Après ces énoncés, plus de cinquante membres du Parlement (Bundestag) ont quitté la salle dans un sentiment de scandale ; l'affaire fit grand bruit et Jenninger dut quitter ses fonctions dès le lendemain dans une atmosphère d'outrage national.

Jenninger est évidemment insoupçonnable de sympathies nazies ; quelles qu'aient été ses intentions (Ensink 1997 suggère qu'il souhaitait s'inscrire comme Allemand dans le poids de l'héritage historique de la nation), elles ne pouvaient être que de communiquer, de manière ultime, sa détestation du Nazisme et de ses crimes. Cependant, la réaction de l'assemblée devant quelqu'un qui, même seulement en apparence, pouvait sembler légitimer le vote pro-Nazi, ne serait-ce que pour mieux le condamner plus tard, est tout sauf surprenante. Jenninger lui-même faisait, nécessairement, la supposition qu'il était évident qu'il se dissociait des propos qu'il représentait, et cependant sa performance s'est trouvée mal contrôlée et ses anticipations au sujet de l'auditoire étaient erronées. Sans doute son intention de se dissocier n'était-elle pas suffisamment évidente, ou même si elle l'était, quelque chose dans le dispositif énonciatif empêchait de faire prévaloir la conscience de cette dissociation sur les sentiments produits par une telle représentation. C'est là le point crucial sur lequel nous souhaitons insister : même si l'auditoire savait que Jenninger n'était pas un sympathisant nazi, le choc, le dégoût, la révulsion que cette performance produisait était impossible à ignorer.

20 A quoi ces sentiments ont-ils tenu? A quoi cette réaction est-elle imputable? Certainement pas aux contenus propositionnels. Il est parfaitement possible de décrire (putativement) les sentiments d'un individu quelconque, fût-ce un pro-Nazi, sans susciter de scandale. D'ailleurs, un an plus tard, un membre important de la communauté juive, Ignatz Bubis, a utilisé plusieurs passages du discours de Jenninger verbatim sans susciter de tels effets. Ainsi donc, ce ne sont pas, au fond, les mots choisis, 
la forme propositionnelle produite, qui ont fait horreur, mais la performance discursive dans son entièreté, dont, en particulier, le choix du style indirect libre, qui ne décrit pas mais agit en quelque sorte par mimétisme. Or le fait de mimer ne relève pas du domaine de la propositionnalité. Le mimétisme produit par le style indirect libre, qui implique un certain degré d'identification tant de la part du locuteur que de celle du destinataire, a ici manifesté une attitude et produit des émotions.

21 Le terme de malentendu ne semble qu'imparfaitement convenir ici. Un malentendu typique relève d'une interprétation différente du contenu propositionnel d'un énoncé ou d'un sous-entendu relativement aux intentions du locuteur, mais il s'agit toujours de matériel propositionnel. Le genre de malentendu qui se produit dans le cas du discours de Jenninger ne suppose absolument pas de divergence au niveau propositionnel; il s'agit plutôt d'un effet spécifiquement émotionnel.

Le «cas Jenninger» suscite un ensemble de questions. Nous laisserons de côté certaines d'entre elles qui concernent le rôle du contexte institutionnel, la position hiérarchique du locuteur, l'arrière-plan général, etc. Nous explorons dans ce qui suit plutôt les processus pragmatiques qui entrent en jeu lorsque la dimension expressive d'une interaction joue un rôle prédominant dans l'interprétation. Dans l'affaire Jenninger, les contenus propositionnels ne sont pas dépourvus de pertinence, mais la dimension émotionnelle prend le pas sur eux, les rendant secondaires ou négligeables. Cet aspect affectif de la communication est la dimension expressive étudiée par Bally. Sa trace la plus directement identifiable dans l'usage du langage est donnée par les « expressifs ».

\section{La dimension expressive}

Lorsque nous parlons de la dimension expressive, nous pensons à des exemples comme (2), (4) ou encore (5) et (6) ci-dessous :

(5) (D'un ton dégoûté) : Cet enfoiré de Thompson a encore insulté la nation entière.

(6) (D’un ton excédé) : Ces enfoirés d'assureurs ont demandé un putain de délai supplémentaire.

Ces deux énoncés contiennent quelque chose d'ineffable, qu'il est impossible de rendre avec des mots et des conditions de vérité, à savoir avec de la signification non-naturelle dans l'acception de Grice. En effet, les contenus propositionnels de ces exemples sont équivalents à, respectivement, (7) et (8), en termes de conditions de vérité, bien que ces derniers ne laissent plus saisir la valeur expressive de (5) et (6).

(7) Horace Thompson a insulté la nation entière.

(8) Les assureurs ont demandé un délai supplémentaire.

On pourrait se demander si enfoiré en (5) et enfoirés et putain en (6) ne feraient pas qu'ajouter des conditions de vérité supplémentaires, à la manière des adjectifs en général. C'est une question délicate : si enfoiré en (5) ajoute la condition Thompson est un enfoiré, cela serait tenable, mais il se trouve que les expressifs ajoutent à cette notion quelque chose de plus : l'exclamation de rage elle-même que ces termes servent ici à 
transmettre, et qui est le composant qui nous intéresse. On le voit mieux encore avec un putain de délai où putain ne peut même plus associer une propriété claire à délai : il ne peut s'agir d'une opposition entre les délais putains et ceux qui ne le sont pas. Il s'agit de toute évidence d'un terme trahissant une attitude émotionnelle, dont la portée est d'ailleurs difficile à établir, dans le sens où cette émotion ne porte probablement pas sur le délai en question mais sur l'entier de la demande formulée par les assureurs. De la sorte, il reste de toute manière un élément d'ordre attitudinal qui résiste irréductiblement à l'analyse propositionnelle. Exprimer des émotions au sujet de quelqu'un en utilisant une épithète comme enfoiré n'est pas la même chose que de décrire quelqu'un comme disposant de la propriété d'être un enfoiré. C'est dans ce sens que les termes expressifs de (5) et (6) peuvent être dits descriptivement ineffables: aucune proposition, ni série de propositions, ne permet de rendre compte de leur effet attitudinal, émotionnel, expressif, spécifique.

D'autres cas de figure nous éclairent sur cette question, et notamment les interjections (dont le statut fait l'objet de débats nourris, cf. Wharton, $2003 ; 2009)$. (10) et (12) cidessous décrivent des états émotionnels, et sont effectivement des assertions évaluables en termes de conditions de vérité, mais par contraste, (9) et (11) expriment ces états de manière directe :

(9) Aïe !

(10) Ca fait mal ! / J'ai mal !

(11) Aha !

(12) Je suis surpris !

Wharton (2003), puis Kleiber (2006 ; 2016), ont montré qu'une interjection comme (9) ne se réduit pas à son contenu propositionnel (10), puisque l'énoncé (13) n'exhibe pas de redondance, au contraire de (14) :

(13) Aïe ! J’ai mal !

(14) J'ai mal ! J'ai mal !

La valeur non-propositionnelle de Aïe! se manifeste encore par l'impossibilité pour l'interjection d'entrer dans le jeu épistémique auquel se prêtent les propositions (observations et exemples originaux en anglais de Wharton, 2003 et de Kleiber, 2016 pour le français, que nous reprenons ici) :

A : Aïe !

B : - *C'est faux ! / Ce n'est pas vrai

A : J'ai mal 
B : - C'est faux / Ce n'est pas vrai

A : Aïe!

B : - ? Je le sais

A : J’ai mal

B :- Je le sais
$\mathrm{A}: \mathrm{Tu}$ as mal ?
B : - (Oui) *Aïe !

La conclusion s'impose : il y a en (9) un élément de contenu irréductible à son contenu propositionnel. Pour l'essentiel, l'hypothèse de Kleiber (2016) est que le cri de douleur manifesté par l'interjection connecte l'impression subjective de douleur à la douleur connue d'autrui dans la collectivité, car le déploiement intonatif doit s'appuyer sur un support verbal, lequel est partagé dans la communauté.

30 Il ressort de cela, et d'autres observations (cf. Kleiber 2016, 123), que les expressifs sont déictiques : ce sont des "symptômes » au sens de Bühler (1934), et ils ressortissent au domaine du montré plutôt que du dit (Wharton 2015). Or ce mode « expressif » implique directement l'émotion, et c'est pourquoi il est crucial pour notre compréhension de la manière dont les effets de communication liés à l'émotion s'interprètent: "le mode exclamatif ou expressif, de manière indexicale, présente ou manifeste déjà l'émotion » (Kleiber, $2006: 21$ ). Les expressifs indiquent la performance d'un certain acte de langage (Wharton 2003; 2015); de la sorte, leur contribution dans la communication est indépendante de la proposition contenue dans l'énoncé dans lequel ils apparaissent (ce qui la rend particulièrement difficile à expliciter). Enfin, les expressifs - et bien évidemment les autres manifestations affectives dans le langage - dépassent le cadre linguistique (ils s'accompagnent volontiers d'autres modes d'expression: gestes, intonation...) et ils ont même quelque chose de non-linguistique.

31 Si la pragmatique issue des travaux de Grice n'envisage rien ou presque au sujet de ces contenus, sans parler de la sémantique formelle, il nous semble que quelques pistes peuvent se dessiner pour une théorie plus anthropologique de la communication. Nous pensons à la pragmatique « cognitive ». 


\section{La pragmatique cognitive et le sens non propositionnel}

32 Le courant parfois appelé pragmatique cognitive, essentiellement né des travaux de Wilson (1973) puis Sperber \& Wilson (1986/95) sous le nom de Théorie de la pertinence (voir aussi Blakemore, 2002 ; Carston, 2001), accorde une importance première aux processus cognitifs qui conduisent le destinataire à découvrir l'intentionnalité du locuteur, son vouloir-dire. Bien qu'elle se soit également concentrée sur les aspects propositionnels de la communication, la question se pose de savoir comment elle peut prendre en considération ses aspects expressifs, descriptivement ineffables. La Théorie de la pertinence part d'hypothèses déjà exprimées par Grice, et notamment celle qui suggère que le sens intentionnel est reconstruit par le destinataire de manière inférentielle. L'approche de la théorie de la pertinence, post-gricéenne, s'éloigne du modèle abductif de Grice, parfois interprété comme conventionnaliste (Bach 2006; Horn 2006; Levinson 2000) et considère que le destinataire applique deux principes fondamentaux inter-reliés (pour davantage de détail, cf. Sperber \& Wilson, 1986/95; Allott, 2013).

33 Premièrement, le principe cognitif de pertinence stipule que le système cognitif humain, de manière générale, est orienté vers la recherche d'informations pertinentes. Ces informations ont la propriété de modifier l'état cognitif de manière significative (l'énoncé mérite d'être traité) mais corrélative à l'effort mental requis pour l'obtenir (décodage et inférence). Ces informations, de nature propositionnelle, correspondent aux effets cognitifs (positifs). Comme les locuteurs savent que leur destinataire recherche des informations pertinentes, ils doivent présenter le stimulus communicatif comme tel, c'est-à-dire comme valant l'effort d'être traité. Ainsi, deuxièmement, le second principe, dit principe communicatif de pertinence, stipule que le fait de présenter de manière ostensive une intention d'informer crée une présomption, chez le destinataire, que le stimulus correspond à ce critère de valoir la peine d'être traité; et même qu'il sera optimalement pertinent. La pertinence est maintenue, qu'il s'agisse d'énoncés porteurs de faibles effets mais demandant de faibles efforts, ou d'énoncés produisant de grands effets malgré des efforts plus importants ; le tout est que l'effet compense aussi largement que possible l'effort de compréhension.

34 Cette présomption de compensation de l'effort par l'effet, c'est-à-dire de pertinence, déclenche le processus interprétatif qui suit un chemin du moindre effort, agrégeant les informations potentielles au fur et à mesure de leur accessibilité jusqu'à obtenir la pertinence optimale. C'est pour cette raison que nous interprétons des contenus implicites, lorsque le sens explicite ne résonne pas suffisamment avec notre arrièreplan épistémique (notre ensemble de croyances et connaissances préalables ou environnement cognitif). C'est aussi pour cette raison que nous ajustons le sens lexical au contexte et que nous enrichissons les formes linguistiques en général, et en particulier les phrases, afin de nous conformer à la recherche d'informations pertinentes. Il convient encore d'ajouter que le contenu propositionnel n'est pas donné clé en mains mais fait l'objet d'une reconstruction ad hoc par le destinataire qui le contextualise. Même les représentations sémantiques exigent un ensemble d'ajustements contextuels, guidés par les mêmes principes, et par la théorie de l'esprit (Sperber \& Wilson, 1998 ; Barsalou, 1987). Il s'ensuit que non seulement les contenus implicites mais également le contenu propositionnel explicite n'est pas un donné transparent du langage mais une 
reconstruction dans les circonstances; ils sont le résultat d'un processus appelé enrichissement pragmatique.

La caractéristique de ces contenus explicites est qu'ils sont annulables sur le plan de la logique pure (ce sont donc des contenus implicites pour Grice) mais ils ne le sont pas de manière plausible pour la psychologie. Ainsi, même si du point de vue logique « Marie a quatre enfants » n'exclut pas qu'elle en ait cinq, il en va pourtant ainsi du point de vue psychologique (le contenu «Marie a exactement quatre enfants» ne peut être l'objet d'une dénégation plausible au sens de Pinker \& al. 2008 et leur rétractation fait intervenir un sentiment de mauvaise foi, $c f$. de Saussure \& Oswald 2009). Dans les circonstances, ce n'est pas une norme conventionnelle ou une maxime conversationnelle qui permet ce résultat, mais la recherche cognitive de pertinence.

36 La manière dont les auteurs de la théorie de la pertinence envisagent de traiter la dimension non propositionnelle du sens passe par la notion de manifesteté (Sperber \& Wilson 2015). Les contenus implicites, appelés implicatures, peuvent être fortement manifestes, s'ils sont indispensables à la pertinence, ou faiblement manifestes, s'ils ne le sont pas. Sperber \& Wilson traitent des contenus non-propositionnels, en particulier ce qu'ils appellent des impressions, notamment dans le contexte littéraire, comme des ensembles d'implicatures faiblement manifestes, une " collection de propositions " (« an array of propositions») relativement indéterminée de sorte qu'il est impossible de fixer précisément lesquelles seront rendues manifestes à un destinataire (d'où la variabilité de l'interprétation d'un individu à l'autre). Leur cas d'école est la métaphore créative (cf. Sperber \& Wilson 2015, 22 et ci-dessous section 7), comme l'énoncé «Juliette est le soleil» dit par le Roméo de Shakespeare, qui diffuse un ensemble indéterminé d'idées relatives au soleil et à l'amour. Cependant, la nature ultime de ces éléments ne peut guère être autre chose que propositionnelle s'il s'agit de «collections de propositions».

37 Nous suggérons donc d'ajouter à ce panorama une notion d'effets émotionnels (cf. Wharton \& Strey, 2017, Briens \& de Saussure, 2018) à proprement parler.

\section{La notion d'émotion et les effets émotionnels}

Tentons de présenter premièrement ce que nous entendons par émotion. Avec Rey (1980) nous considérons que les émotions se distinguent des sensations ou des sentiments par le fait qu'elles impliquent une interaction entre un élément cognitif, d'une part, et des éléments physiologiques et qualitatifs d'autre part. Si nous prenons l'exemple de la peur, cette émotion est caractérisée par l'interaction entre une sensation - l'élément physiologique, dont fait partie la sécrétion d'épinéphrine, un neurotransmetteur associé au changement de rythme cardiaque et respiratoire, et de cortisol, qui augmente l'attention et la mémoire à court terme (tout en impactant négativement la capacité analytique) -, un sentiment - l'élément qualitatif, le sentiment physique d'être effrayé, qui s'accompagne d'un ensemble de comportements cohérents avec ce sentiment -, et un élément cognitif - une croyance d'être en danger pour une certaine cause, d'être dans une situation non souhaitable. Dans cette perspective, alors que les états émotionnels impliquent de manière cruciale des éléments cognitifs autant que qualitatifs et physiologiques, les sentiments et les sensations ne l'exigent pas ${ }^{3}$. Dans notre acception, «ressentir " une émotion n'implique pas les processus cognitifs qui gèrent les états épistémiques et les autres aspects représentationnels. Les sentiments, 
après tout, n'ont pas besoin d'être conscients. Avoir un sentiment que le temps se gâte, ou que l'on n'a pas réussi un examen, présuppose certes un état épistémique - une croyance à propos de ces choses - mais est en soi autre chose, que "croire " ou «penser » ces choses. Avoir le sentiment que quelqu'un est attiré par vous, ou que deux personnes parlent de vous en secret, ne se réduit pas à croire ces choses. Et plus encore : on peut avoir une angoisse indéterminée, c'est-à-dire sans même pouvoir identifier une quelconque croyance angoissante.

39 Bien que la terminologie diffère, notre conception présente des points de convergence qui la rend compatible avec les hypothèses fondamentales de la théorie de l'évaluation cognitive (appraisal theory, Ellsworth, 2013 ; Frijda, 2007 ; Lazarus, 1991 ; Deonna and Teroni, 2012): les émotions consistent en plusieurs composants et impliquent une évaluation qui cause la réaction (bien que pour nous, comme nous l'exposons plus loin, les effets émotionnels jouent un rôle causal). Cette conception s'articule également avec la perspective de Cosmides \& Tooby (2000/08), qui considèrent que l'esprit humain dispose d'une architecture neuronale propre à son espèce et qui a évolué en réponse à des problèmes adaptatifs. Ils définissent l'émotion comme un programme cognitif superordonné dont la fonction est de réguler ou de mobiliser des sous-programmes cognitifs responsables de la perception et de l'attention, l'identification des buts, la collecte d'informations, les changements physiologiques et des types spécialisés d'inférences.

40 Depuis au moins David Hume, on considère que la rationalité seule ne suffit pas à motiver l'action de raisonner. Cette motivation, à l'en suivre, ne peut provenir que des " passions »; la cognition et l'affect travaillent bien entendu ensemble de manière complexe. Pour le dire plus précisément, citons Greenspan (2002 : 206) :

(...) [L]es émotions peuvent fonctionner comme des causes permettant la formation de décisions rationnelles (...) dans la mesure où elles dirigent l'attention vers certains objets de pensée et l'éloignent d'autres objets de pensée. Elles servent à augmenter la mémoire et à réduire l'ensemble des options praticables à un ensemble gérable, adapté à des prises de décisions «rapides et brutales » ['quick and dirty']. (Greenspan, $2002: 206$, notre trad.).

41 Les stimuli émotionnels génèrent des processus cognitifs en contraignant la construction du contexte ${ }^{4}$ dans lequel les fondements informatifs et propositionnels de l'état émotionnel, par exemple que le locuteur est anxieux, effrayé, fâché, excité, etc., sont interprétés par le destinataire. C'est dans ce sens que nous comprenons Greenspan quand il exprime l'idée que les émotions limitent l'ensemble des options praticables. Les émotions ont un impact sur l'accessibilité, ou la manifesteté de ces différentes options et elles le font en dessous du niveau de conscience.

42 Le domaine de la Théorie de la pertinence est traditionnellement celui de la «communication ostensive (intentionnelle) inférentielle». Or les comportements spontanés et naturels, tels que les sourires, les frissons etc., peuvent être exhibés de manière parfaitement délibérée, "non-naturelle", dans une mesure plus ou moins grande $^{5}$, ce qui conduit de tels comportements à appartenir au domaine de la communication intentionnelle. Toutefois, de même que les énoncés sont des complexes de comportements naturels et non-naturels, l'interaction humaine en général implique des processus situés en dessous du niveau de conscience. Il ne s'agit pas ici d'une posture nouvelle :

A chaque instant, nous communiquons des informations au sujet de notre état émotionnel, nos attitudes, et nos évaluations au sujet de ce à quoi nous sommes 
actuellement confrontés (...). Nous produisons la plupart de nos indices nonverbaux de manière intuitive, sans conscience phénoménologique. (Liebermann, $2000: 111$, notre trad.).

Pour préciser ce que nous appelons des effets émotionnels, relevons d'abord que la communication émotionnelle agit sur un ensemble de niveaux différents. Les interjections, l'expression du visage et les tons de voix affectifs apportent des informations de haut niveau en ce sens qu'elles sont au sujet de l'énoncé dans son ensemble, et favorisent des effets cognitifs positifs. Mais ce qu'apporte la communication émotionnelle ne s'y réduit pas; il faut donc introduire une notion d' effets émotionnels. Un effet émotionnel, pour nous, sera la réaction interprétative suscitée par la manifestation directe d'une émotion (et non sa description).

44 Un «expérienceur» du type de sensations ou de sentiments évoqués ci-dessus, typiquement associés à la peur, devient automatiquement hyper-alerte. Dans cet état, il accordera une attention élevée à des perceptions qu'il ne remarquerait peut-être pas dans d'autres circonstances. Une fois que la cognition est impliquée, c'est tout un nouvel ensemble de buts qui seront définis, et ce sont certains processus inférentiels qui deviendront prioritaires. Selon Wharton \& Strey (2017), ces processus sont activés par des procédures émotionnelles.

45 Le traitement des effets émotionnels implique une idée d'expérience intime et personnelle plutôt que de représentation et de gestion d'information conceptuelle. Cette expérience est décrite par Sperber \& Wilson sous le terme de "schémas d'activation, qui ne peuvent se décrire de manière appropriée par une notion de fixation d'une croyance » (Sperber \& Wilson, 2015 : 139, notre trad.). Ils n'engagent pas une recherche de pertinence au sens classique mais agissent typiquement comme des précurseurs de cette recherche si nécessaire. Dans certains cas, comme dans l'affaire Jenninger discutée ci-dessus, les effets émotionnels constituent l'essentiel de ce qu'on retire du discours. Dans d'autres cas, ils servent de point de départ à une recherche d'effets cognitifs positifs. Ainsi ce n'est pas que les effets émotionnels n'auraient rien à voir avec la dérivation d'effets cognitifs, mais plutôt que les deux dimensions interagissent ; cela se produit notamment lorsque le destinataire infère qu'une émotion a été montrée de manière délibérée par un locuteur. Cependant, un énoncé peut produire ses effets communicatifs émotionnels sans devoir nécessairement engendrer une recherche supplémentaire de pertinence par l'inférence d'effets proprement cognitifs.

46 Lorsque la manifestation d'une émotion n'est pas ostensive, au sens d'intentionnelle et dirigée vers un interprète, l'interlocuteur, ou le témoin d'une scène où de l'émotion est présente - par exemple Jacques rougissant au contact de Mia - saisit cette émotion dont il peut ensuite tirer toutes sortes de conséquences dans les circonstances. Mais si un état émotionnel est perçu comme étant ostensif - délibérément montré à un destinataire - les choses sont un peu différentes: le destinataire recherche non seulement - éventuellement - les raisons de l'émotion mais aussi les raisons pour lesquelles l'état émotionnel a été rendu ostensif. Autrement dit, il s'agit de comprendre pourquoi le porteur d'un état émotionnel, par exemple le locuteur d'une interjection, a l'intention délibérée de lui faire savoir qu'il est porteur de cet état émotionnel déterminé. La réponse se fait à nouveau en termes d'inférences, de nouveaux effets cognitifs positifs, pour lesquels l'état émotionnel contraint le contexte interprétatif, ou, 
pour le dire autrement, l'état émotionnel permet d'accéder à des hypothèses qui serviront dans le contexte interprétatif.

Dans une scène fameuse du film Zorba le Grec (M. Cacoyannis, 1965), le héros, Zorba, travaille à l'intérieur de la galerie d'une mine vétuste lorsqu'une partie de la voûte s'écroule avec fracas. L'entrée de la galerie est soudainement obscurcie par un épais nuage de poussière. Avec angoisse, son ami crie : «Zorba! ». Tous les ouvriers qui sont présents et attendent autour de la galerie sont immédiatement alertés par ce cri qui les met dans un état préparatoire à l'action. Du ton de la voix de son ami, Zorba interprète immédiatement son état émotionnel. Cependant, cet état émotionnel est présenté ostensivement, de sorte que la proposition épistémique - son ami est terrifié à l'idée qu'il soit blessé - lui devient accessible en même temps que l'idée que le locuteur a eu l'intention de rendre ce fait manifeste à son intention. Le cri, en quelque sorte, témoigne d'attentes du locuteur, à savoir une forme de réaction du destinataire et peut-être des témoins (destinataires indirects), et a donc l'intention que cette intention soit reconnue. En retour, cette proposition peut avoir un ensemble de conséquences en termes de prémisses entrant dans une inférence. Dans le film, elle a une conséquence particulière, qui est que l'ami de Zorba pense qu'il a été imprudent en allant éprouver le toit de la mine. C'est une inférence qui ne peut être dérivée que comme hypothèse, sur la base de l'effet émotionnel lui-même, pris comme prémisse. Et c'est précisément cette hypothèse à laquelle répond Zorba lorsqu'il ressort indemne en énonçant d'un ton agacé : "Quoi?». Cette réponse communique que Zorba choisit d'ignorer l'état émotionnel, sans quoi il lui aurait fallu objecter explicitement à l'idée qu'il n'a pas été assez prudent. Au contraire, sa réponse communique que son ami n'aurait pas dû s'inquiéter et que son état émotionnel était donc inapproprié vis-à-vis de la situation. Ainsi, l'effet émotionnel a orienté le contexte interprétatif et les attentes de pertinence. Les émotions jouent un rôle si important dans l'interaction humaine qu'il semble raisonnable d'admettre qu'ils sont aussi courants dans la communication linguistique que les effets cognitifs. Les effets émotionnels sont centraux dans la communication humaine et pourraient même avoir joué un rôle décisif dans l'évolution de la communication linguistique, par le passage de l'expression spontanée à l'interjection produite de manière délibérée, avec la conscience, pour le locuteur, que le caractère délibéré - ostensif - du stimulus sera non seulement reconnu mais traité inférentiellement (c'est la suggestion de Grice, 1989 : 293-297).

\section{Effets émotionnels et créativité expressive}

Dans de nombreux cas, les effets émotionnels permettent au communicateur de dépasser les limitations du sens propositionnel et ouvrent ainsi à la créativité expressive. L'ironie est un cas particulièrement intéressant car elle lie directement des attitudes descriptivement ineffables, des contenus émotionnels, des propositions et des effets de face dans l'interaction, or, et c'est important pour ce qui concerne la créativité langagière, l'ironie n'est pas linguistiquement normée. Elle est purement inférentielle, interprétée uniquement par l'identification d'une incongruité entre ce qui est énoncé et les circonstances de l'énonciation: il n'y a pas d'indicateurs linguistiques de l'ironie, tout comme il n'y a pas d'indicateurs linguistiques du style indirect libre. L'ironie comme le style indirect libre sont (à tout le moins pour la Théorie de la pertinence en ceci inspirée par Ducrot 1984) des cas de citation ou de mention sans indication explicite 
de la citation (Sperber \& Wilson 1995). L'ironie n'est détectée qu'à partir de l'âge de 6 ou 7 ans lorsque les enfants, équipés d'une théorie de l'esprit suffisante, peuvent envisager une intention méta, une instrumentalisation de l'énonciation afin de communiquer autre chose que le contenu incongru, et entrer dans un travail heuristique inférentiel qui permet d'incorporer l'attitude dissociative du locuteur vis-àvis du contenu exprimé.

50 Cependant, l'intéressant, ici, est que ce qui est communiqué en propre par l'ironie n'est pas propositionnel, mais, au contraire, de nature émotionnelle. Et d'ailleurs il est facile de constater l'effet, souvent désagréable, produit par une ironie ; un tel effet, cela va de soi, est la réponse par une émotion à un effet communicatif émotionnel (nous entrerons dans la section suivante plus en détail dans la nature de ces effets et du processus interprétatif qui les concerne). Pourquoi dire que l'effet ironique n'est pas propositionnel ? Parce qu'une paraphrase d'énoncé ironique, qui contiendrait tous les éléments de sens produits par l'analyse, fait disparaître toute dimension ironique (de Saussure \& Schulz 2009). Ainsi, l'ironie en tant que telle, de manière essentielle, échappe à la propositionnalité. Si (15), prononcé ironiquement alors qu'il pleut, correspond à quelque chose comme (16) (parmi d'autres possibilités imaginables), on constate que (16) a perdu toute trace d'ironie, et d'ailleurs ne produit pas du tout les mêmes effets. Nous sommes donc dans un cas parfaitement comparable à celui de Aïe!:

(15) Beau temps pour une promenade !

(16) Tu as dit tout à l'heure qu'il ferait beau, or il pleut, et donc ta prédiction est rétrospectivement ridicule (d'après de Saussure \& Schulz, 2009 : 402).

51 Pour de Saussure \& Schulz (2009), les attitudes portées par l'ironie se rapportent au monde du savoir " procédural », par opposition au savoir « déclaratif » : c'est le type de différence qu'on évoque généralement pour montrer qu'on ne peut pas enseigner à faire $\mathrm{du}$ vélo avec succès uniquement en transmettant des informations propositionnelles; il faut en effet recourir à la procéduralité de l'expérience, qui est donc ineffable.

52 Ceci nous permet de revenir au cas de Jenninger. Comme pour l'ironie, l'énoncé de Jenninger est citationnel, puisqu'il est au style indirect libre, qui est une forme de mention. Cependant il ne comporte pas l'attitude dissociative définitoire de l'ironie. Il peut certes arriver que style indirect libre et ironie se mêlent, par exemple lorsque Flaubert déploie en direct, par le recours au style indirect libre, les émotions diverses et variées de Frédéric dans l'Education sentimentale. L'ensemble subtil d'effets attitudinaux produits par le style indirect libre est relativement indistinct, produisant plutôt des impressions d'attitudes que des conclusions claires à leur sujet - c'est une ressemblance avec les métaphores créatives mais c'est aussi une différence importante avec l'ironie. Le cas Jenninger montre que de tels effets émotionnels, ineffables, non propositionnels, produisent une réponse expérientielle plutôt que représentationnelle; ils n'entrent pas nécessairement dans une chaîne inférentielle et donc ne donnent pas nécessairement lieu à des représentations de nature propositionnelle. Le problème provoqué par le discours de Jenninger tient au fait qu'une interprétation ironique, et donc clairement dissociative, est rendue impossible par les circonstances de l'énonciation et le contexte de manière générale; et ce sont donc d'autres émotions qui seront générées au titre de réponses psychologiques chez les destinataires. Or, l'interprétation au style indirect 
libre, comme pour tout énoncé rapporté, ne permet pas de statuer sur l'endossement par le locuteur des contenus qu'il rapporte (voir Morency, Oswald et de Saussure 2008). En ce qui concerne le cas Jenninger, il se trouve qu'il existe pour l'auditoire quelques éléments saillants qui non seulement empêchent une interprétation ironique mais ouvrent à une interprétation possible pour laquelle le locuteur éprouve une certaine sympathie pour la personne dont il rapporte les propos. Ces éléments sont les suivants : a) le Président du Bundestag ne peut en aucun cas se moquer du représentant ordinaire évident de la nation, à savoir l'Allemand " ordinaire ", et donc il ne peut pas faire acte d'ironie ; b) il produit un discours (imaginaire) pro-Nazi, ce qui est choquant en toutes circonstances mais d'autant plus dans la commémoration de la Nuit de Cristal) ; c) ce faisant, il fait le portrait de l'Allemand «ordinaire» des années trente comme drastiquement antisémite et pro-Nazi et suggère qu'il s'agit-là de la composante essentielle de l'opinion publique de ce temps. Du fait de ces éléments, l'auditoire ne peut inférer que le locuteur se dissocie clairement des propos tenus. Bien évidemment, un ensemble de raisons d'arrière-plan, comme le parcours personnel de Jenninger, permettraient de lever le doute, mais les éléments rendus manifestes par le locuteur lui-même restent en retrait à ce sujet. L'auditoire ne peut que conclure que non seulement Jenninger a été pragmatiquement incompétent ce jour-là, ce qui est inattendu de la part d'un politicien de haut rang, mais, quelque vaguement que cela fût, sympathique à l'égard du sympathisant nazi ordinaire - position bien entendu choquante et inacceptable.

53 Dans ce type de cas, il faut souligner que l'effet émotionnel survient sans que le locuteur en ait eu la moindre intention (ici, bien au contraire), mais parce qu'il a échoué à prédire que ces effets surviendraient à cause de son comportement linguistique (et donc à les empêcher). L'effet émotionnel a complètement stoppé toute possibilité de traiter analytiquement les autres aspects, propositionnels, explicites et implicites, cognitifs, du message: l'auditoire n'a que faire à ce stade des réelles intentions du locuteur.

54 Il ne s'agit là que d'un cas parmi d'autres où l'esprit applique des heuristiques qui ne travaillent pas avec des propositions ou pas uniquement avec des propositions. Le cas des sophismes et argumentations fallacieuses est devenu un classique, puisque la recherche tend à considérer leur potentiel succès sur la base de l'exploitation d'heuristiques non analytiques ou de « biais cognitifs " (Maillat \& Oswald, 2009) ${ }^{6}$. Mais il suffit de songer à ce qui se produit avec les insultes, qui cette fois sont nécessairement intentionnelles, pour comprendre que la réaction à ces effets ne se place pas sur le plan argumentatif (propositionnel) mais purement affectif, assaillant l'interlocuteur d'effets émotionnels qui l'orientent vers d'autres types d'interactions. Tout ceci nous ramène au fait que les effets émotionnels monopolisent des ressources et affectent la chimie neurologique, orientant les individus vers l'action.

55 Parmi les ressources typiques de l'expressivité émotionnelle se trouvent les métaphores créatives. Une métaphore comme «Juliette est le soleil » (dans le Roméo et Juliette de Shakespeare, commentée par Sperber \& Wilson 2015, 22) est simplement imparaphrasable sans la perte de la dimension - quelle qu'elle soit - qui la rend expressive, puissante et pleine de « sens ». Mais nous proposons d'ajouter que, même si diverses propositions peuvent être activées par la métaphore créative (et être faiblement manifestes), elles activent aussi et surtout un ensemble de traces mémorielles personnelles, d'expériences intimes, des sentiments imaginaires, qui 
articulent à la fois ce qui est produit par l'amour dans le sens particulier de l'amour passionnel de Roméo pour Juliette, manifeste dans le contexte et qui correspond à un concept spécifique à l'intérieur de l'ensemble de tous les concepts d'amour imaginables, et par le soleil. Hormis des propriétés purement conceptuelles comme la chaleur, la lumière, l'unicité, etc., elles font donc émerger des sentiments qui y sont associés, les bienfaits de la chaleur solaire, l'illumination du monde autour de soi, la hauteur dans le ciel, etc. En d'autres termes, ce sont bien des affects, plutôt qu'une collection de propositions, qui motivent réellement de telles métaphores.

\section{Du partage des émotions à leur pertinence par simulation}

Il s'agit alors de partager des émotions, or ce n'est pas exactement la même chose que de les communiquer. Les décrire par des phrases, et donc par du matériau propositionnel, permet de les communiquer, mais les évoquer directement, c'est-à-dire les rendre manifestes par l'expressivité, permet plutôt de les partager. La différence peut s'exposer ainsi : communiquer, dans un sens restreint, consiste à rendre accessible à autrui, ou manifeste, des informations, au sujet desquelles on peut ensuite se positionner de manière évaluative (on peut les trouver justes ou fausses, intéressantes ou non, etc.). Mais partager un état mental, une émotion, consiste à rendre manifeste à autrui cet état mental comme un fait qui se situe hors de l'évaluation analytique. Ce partage pourrait être l'effet d'une forme de simulation d'autrui, ce qui n'est pas requis dans la communication d'informations.

57 Si nous reprenons le cas de la métaphore Juliette est le soleil, on peut admettre un effet de simulation des émotions intimes de Roméo; il est clair que les métaphores sont bien en un sens des descriptions, mais elles peuvent déclencher des effets émotionnels qui résonnent avec les émotions intimes d'autrui, que ce soit par l'activation de traces mémorielles personnelles ou par l'imagination de se retrouver soi-même dans la position de Roméo et donc de simuler les sentiments qu'il pourrait avoir. Cette simulation, vraisemblablement, est au fond le véritable effet produit par de telles métaphores, et la question reste ouverte de savoir si les effets émotionnels en général ne seraient pas après tout de nature simulative. Notre point de vue est qu'il en va de même avec des choses aussi triviales que Aïe! ou les autres expressifs. Aïe! activerait un effet de simulation, au contraire de J'ai mal, ce qui pourrait expliquer leurs différences de comportement linguistique. Il en va évidemment de même également avec le cri « Zorba! », etc.

58 D'autres métaphores créatives, cependant, peuvent très bien ne susciter aucun effet émotionnel identifiable de ce genre. Il ne s'agit donc pas pour nous d'affirmer que toutes les métaphores, ou que tout ce qui est imparaphrasable ou ineffable, suscite des effets émotionnels ou même attitudinaux comme peut le faire l'ironie. Quand Flaubert dit de Leconte de Lisle que "Son encre est pâle », il n'est pas clair qu'il faille supposer un quelconque effet émotionnel. Cela dépend de la manière dont un individu réagira à la touche péjorative de ce jugement.

59 Nous voudrions en outre suggérer que les métaphores créatives ne sont pas exceptionnelles en ce qui concerne leur mode de déclenchement d'effets émotionnels. Ces effets ont, de manière générale, à voir avec la manière dont les affects qui sont 
rendus manifestes par un acte ostensif (de parole) provoquent une réponse qui implique une dimension expérientielle: le destinataire trouve dans sa propre expérience, ou dans l'imagination d'expériences possibles, les éléments qui le conduiront à simuler l'expérience sous-jacente communiquée, à savoir l'effet émotionnel.

60 Les métaphores, ainsi qu'un ensemble d'autres effets poétiques, sont courants dans la littérature et bien sûr la poésie. Si nous suivons l'idée que les effets émotionnels suscitent une réponse expérientielle, alors la compréhension d'un poème, sans s'y réduire, suppose la mise en relation de ce que les énoncés rendent manifeste et des sortes d'équivalents dans notre mémoire ou notre imagination expérientielle, et s'ancre donc dans notre vie affective. L'interprétation de la poésie est largement au sujet de la manière de faire sens de notre expérience intime, du sentiment que des états émotionnels et mentaux semblables aux nôtres sont partagés par d'autres, et que nous sommes potentiellement appelés à partager ceux que nous n'avons pas encore connus directement.

61 Le sentiment d'être en colère, ou d'être ému, ou d'être passionnément amoureux, est étranger à quiconque n'a jamais éprouvé de tels affects. Les jeunes enfants peuvent comprendre les histoires d'amour de manière rationnelle mais y sont généralement peu sensibles, au point qu'il n'est pas rare qu'ils s'étonnent que les adultes essaient de communiquer au sujet de l'extrême sophistication des rapports amoureux. Vient cependant un âge où des prémisses liés à des sentiments vaguement de cet ordre leur ouvre la porte pour imaginer et simuler des situations amoureuses - c'est le cas par exemple des hérö̈nes de romans classiques qui se nourrissent de lectures sur les princes charmants en attendant le leur. Non que ces lectures les renseignent efficacement sur les rapports amoureux, et probablement bien au contraire, mais ce n'est pas là le sujet : ce qui se produit est que leur capacité imaginative suscite chez de tels lecteurs des effets émotionnels. Et bien sûr, à plus forte raison dans un monde où les rapports amoureux sont plus faciles, comme dans la société contemporaine. Ainsi, le type d'état émotionnel que l'on éprouve dans la relation amoureuse peut être partagé ; les effets émotionnels ne sont donc pas pertinents au sens technique de la théorie de la pertinence, mais ils sont malgré tout pertinents d'une manière importante et différente, dans le sens où, virtuellement sans effort, ils activent des remémorations expérientielles et suscitent de manière puissante la simulation d'expériences nouvelles.

62 Comme les effets émotionnels ne font pas l'objet d'une reconstruction de signification au sens où les descriptions le font, nous suggérons qu'ils sont communiqués de manière naturelle (dans le sens de Grice), même si une forme de convention linguistique s'applique, avec plus ou moins d'importance, dans leur compréhension.

63 Il reste à revenir brièvement sur deux points importants. Le premier concerne la manière dont il est possible d'intégrer les effets émotionnels dans la théorie de la pertinence qui suppose un seuil d'équilibre entre un certain effort et un certain effet. Le deuxième concerne l'interaction entre les effets cognitifs et émotionnels.

64 Une manière d'envisager l'intégration des effets émotionnels dans le mécanisme de traitement de la communication proposé par la théorie de la pertinence consiste à poser la question du seuil de pertinence en termes de validation expérientielle et non strictement informationnelle. Cela est évidemment spéculatif, mais il s'agirait de dire que la pertinence d'un effet émotionnel est atteinte lorsque la réponse expérientielle est elle-même atteinte, et cela de la manière la plus directe qui soit. Il est possible 
d'obtenir une réponse émotionnelle à partir d'une description, bien entendu, mais alors l'accès à la réponse émotionnelle doit d'abord passer par une interprétation en termes propositionnels, ce qui la rendra moins immédiate, plus demandeuse d'efforts (mais probablement plus riche en termes d'effets cognitifs à proprement parler). On peut également imaginer que l'évocation d'une émotion pour un destinataire qui ne trouve pas de réponse expérientielle à ce stimulus verbal, ni ne parvient à imaginer une forme de simulation, lui sera obscure. Il sera démuni et ne parviendra pas à extraire l'effet émotionnel, le stimulus se trouvant alors dépourvu de pertinence pour cet individu qui ne peut accéder à l'effet émotionnel.

65 En ce qui concerne les rapports entre effets émotionnels et effets cognitifs, il nous semble que plusieurs situations peuvent se rencontrer.

66 Nous connaissons l'effet cognitif «pur » auquel s'intéressent presque exclusivement les traditions formelles. Il est plus difficile de statuer sur l'existence d'effets émotionnels purs, dans le sens où, comme nous l'avons dit plus haut, un affect suscite normalement un fonds épistémique sans lequel il ne peut avoir de sens, mais nous laisserons ce point en suspens. Les cas mixtes sont les plus intéressants : un énoncé descriptif peut bien sûr susciter une forme d'enrichissement émotionnel; ainsi de l'angoisse partagée que l'on peut ressentir si un locuteur nous fait part de manière descriptive d'une angoisse que nous pouvons concevoir. A l'inverse, et comme le montre l'exemple du cri dans Zorba, les effets émotionnels sont propres à susciter un enrichissement cognitif qui leur donnera toute leur motivation sur le plan cognitif. De manière plus générale, les expressions du visage, les intonations affectives et même les interjections dans certains cas déclenchent des explicatures de haut niveau - qui portent sur l'énonciation - et conduisent à des implicites divers, soit en favorisant une conclusion déterminée, soit en altérant marginalement la force ou la saillance d'une collection de conclusions moins déterminées - des effets cognitifs.

67 Il y a donc deux canaux en quelque sorte parallèles de la communication: le canal propositionnel, ou déclaratif, qui produit des effets cognitifs, et le canal non propositionnel, ou procédural, qui produit des effets d'une autre nature, parmi lesquels les effets émotionnels (nous laissons ici de côté une autre dimension de la procéduralité qui concerne des cas d'inférences spécifiques encodées spécifiquement par certains éléments grammaticaux, $c f$. Blakemore 2011).

68 Pour nous, les deux canaux peuvent entrer dans le jeu inférentiel et susciter des effets tant de nature cognitive-propositionnelle que de nature émotionnelle. Par exemple, il va de soi que J'ai mal peut donner lieu à des effets émotionnels, par le résultat empathique produit ex post traitement par la théorie de l'esprit, tout autant que cet énoncé donnera lieu à des inférences propositionnelles. Il en va de même, mais à l'inverse, pour Aïe!: la particularité de ce dernier cas est simplement qu'il transporte ipso facto un effet émotionnel, lequel peut, ou non, donner lieu à des représentations et des inférences, donc des effets cognitifs : l'effet émotionnel est alors direct et n'est pas le résultat d'une évaluation qui suit une description.

69 S'il est vrai que ces deux canaux se nourrissent mutuellement quand les deux types d'effets émergent, et échangeant de l'information au cours de l'interprétation d'un stimulus, cela signifie que l'interprétation fait intervenir simultanément, ou dans un ordre quelconque déterminé selon les différents cas de figure ci-dessus, non seulement une procédure de recherche de pertinence au sens cognitif, contrôlée par ce que la littérature appelle le module de lecture de l'esprit (mindreading module) ou la théorie de 
l'esprit, et une procédure d'accès aux états émotionnels propre à générer des effets émotionnels, dont nous suspectons qu'elle fait intervenir plutôt des dispositifs simulatifs.

70 Une personne dont les procédures émotionnelles sont hautement activées sera davantage susceptible de voir ses états émotionnels reconnus et sélectionnés comme pertinents par autrui (Wharton, 2009, 2015). Ces procédures sous-attentionnelles et non intentionnelles nous conduisent à saisir les états émotionnels, qu'ils soient communiqués de manière ostensive ou non-ostensive. En tout état de cause, ils ne surviennent pas par inférence (dans le sens usuel d'une forme de raisonnement) mais par une heuristique différente. Ressentir l'état émotionnel d'autrui ne se fait pas par le biais d'un algorithme ou de déductions. Ces états émotionnels sont saisis de manière directe et immédiate lorsqu'un individu recourt aux moyens adéquats : expressifs ou autres formes linguistiques chargées affectivement. Ceci n'est pas neuf : il y a une large littérature sur la contagion émotionnelle en psychologie (voir par exemple Hatfield \& al. 1994), qui admet que dans ce cas, non seulement de l'information au sujet des états émotionnels est transmise, mais en réalité et avant tout, ces états eux-mêmes.

\section{Conclusion}

71 Dans cet article, nous avons tenté de dégager quelques pistes en vue d'intégrer la communication expressive, qui concerne les affects, dans le cadre général des approches dominantes, formelles, du langage. Nous leur adressons le reproche de s'être largement désintéressées de tout ce qui concerne la dimension non strictement propositionnelle de la communication, à l'exception de quelques études sporadiques sur certaines propriétés structurelles d'expressions linguistiques isolées (interjections, insultes), et à l'exception, beaucoup plus notable, de travaux récents dans une théorie pragmatique d'orientation cognitive, la théorie de la pertinence de Sperber \& Wilson (1986/1995). Nous élaborons une notion d'effets émotionnels à la suite notamment de Wharton (2009) que nous lions à la notion de "contenus descriptivement ineffables " abordés dans la théorie de la pertinence (Blakemore, 2011 en particulier) et celle d'« impressions » (Sperber \& Wilson, 2015).

72 Notre approche, qui s'inscrit dans le développement des théories de l'émotion comme évaluation (appraisal theory), considère que certains effets cognitifs sont déclenchés par des réactions émotionnelles, et qu'à l'inverse, certains effets émotionnels sont déclenchés par des effets cognitifs; nous suggérons cependant que les effets émotionnels peuvent également atteindre seuls leur pertinence. Cette dernière, en ce qui concerne les effets émotionnels, est atteinte par une procédure heuristique propre qui produit une réponse mentale de type expérientielle et non représentationnelle, que ce soit par l'accès à des traces mémorielles ou par la capacité imaginative.

73 Par l'accès à la capacité imaginative des individus, par la simulation que suscite le partage linguistique des émotions, ce dernier permet de dépasser les limitations formelles de l'informativité et ouvre la porte à la créativité linguistique d'une manière très particulière, qui s'exprime probablement le plus clairement dans la poésie et la littérature par le biais de la figuration et de la non-littéralité. 
74 Cette étude a pour objectif d'offrir un panorama général de cette question sous l'angle pragmatique, qui devrait bien entendu faire l'objet de considérables recherches ultérieures.

\section{BIBLIOGRAPHIE}

Allott, N. (2013) Relevance Theory, in Capone, A., Lo Piparo, F.\& M. Carapezza (eds.), Perspectives on Pragmatics and Philosophy, Berlin/New York: Springer, p. 57-98.

Bach, K. (2006) The excluded middle: semantic minimalism without minimal propositions, Philosophy and Phenomenological Research, 73, p. 435-42.

Bally, C. (1905) Précis de Stylistique, Genève: Eggiman.

Barsalou, L. (1987) The instability of graded structure: implications for the nature of concepts, in Neisser, U. (ed) Concepts and conceptual development: Ecological and intellectual factors in categorisation, Cambridge: Cambridge University Press, p. 101-140.

Benveniste, E. (1970) L'appareil formel de l'énonciation, Langages, 17, p. 12-18.

Blakemore, D. (2002) Relevance and Linguistic Meaning: The Semantics and Pragmatics of Discourse Markers, Cambridge: Cambridge University Press.

Blakemore, D. (2011) On the descriptive ineffability of expressive meaning, Journal of Pragmatics, 43 , p. 3537-3550.

Briens, S. \& L. de Saussure (2018) Les fonctions discursives et narratives des émotions. Pour un nouveau champ de recherche « littérature et émotions ", Revue de littérature comparée, 2018-1, p. 67-82.

Bühler, K. (1934) Sprachtheorie. Die Darstelllungsfunktion der Sprache, Iena: Gustav Fischer Verlag (2e éd. Stuttgart, 1965).

Carston, R. (2001) Thoughts and Utterances, Oxford: Blackwell.

Cave, T. \& D. Wilson (2018) (eds) Reading beyond the code, Oxford: Oxford University Press.

Cosmides, L. \& J. Tooby (2000) Evolutionary psychology and the emotions, in Lewis, M. \& J. Haviland Jones (eds) Handbook of Emotions, New York: Guilford, p. 91-115 (3e éd. 2008).

Damasio, A. (2006) L'Erreur de Descartes. La raison des émotions (1e éd. 1994), Paris: Odile Jacob.

Deonna, J. \& F. Teroni (2012) The Emotions: a philosophical introduction, London: Routledge.

Dezecache, G., Mercier, H. \& T. C. Scott-Phillips (2013) An evolutionary approach to emotional communication, Journal of Pragmatics, 59, p. 221-233.

Ducrot, O. (1972) Dire et ne pas dire, Paris: Hermann.

Ducrot, O. (1984) Le dire et le dit, Paris: Minuit.

Ellsworth, P. C. (2013) Appraisal theory: Old and new questions, Emotion Review, p. 125-131. 
Ensink, T. (1997) Collective misunderstandings due to misframing: The cases of Orson Welles (1938) and Philipp Jenninger (1988), in Rauch, I. \& G. Carr (eds) Semiotics around the World: Synthesis in Diversity, Berlin: De Gruyter, p. 1131-1134.

Frijda, N. H. (2007) The Laws of Emotion, Mahwah: Erlbaum.

Gigerenzer, G. \& P. Todd (1999) Fast and frugal heuristics: the adaptive toolbox, in Gigerenzer, G., Todd, P. \& the ABC Research Group, Simple Heuristics That Make Us Smart, Oxford: Oxford University Press.

van Ginneken, J. (1907) Principes de Linguistique Psychologique, Paris: Rivière.

Greenspan P. (2002) Practical reasoning and emotion, in Mele A. \& P. Rawlings (eds) Rationality, New York: Oxford University Press.

Grice, H.P. (1957) Meaning, Philosophical Review, 66, p. 377-388.

Grice, H. P. (1989) Studies in The Way of Words, Cambridge: Harvard University Press.

Hatfield, E., Cacioppo, J. \& R. L. Rapson (1994) Emotional Contagion, Cambridge: Cambridge University Press.

Horn, L.R. (2006) The border wars: a neo-Gricean perspective, in Von Heusinger, K. \& K. Turner (eds) Where Semantics Meets Pragmatics: The Michigan Papers, Oxford: Elsevier, p. 21-48.

Kleiber, G. (2006) Sémiotique de l'interjection, Langages, 161, p. 10-23.

Kleiber, G. (2016) Du cri de douleur au signe de douleur : l'interjection Aïe, Synergies Pays Scandinaves, 11/12, p. 113-133.

Lazarus, R. S. (1991) Emotion and adaptation, New York: Oxford University Press.

Levinson, S. (2000) Presumptive Meanings, Cambridge: MIT Press.

Lieberman, M. (2000) Intuition: A social-cognitive neuroscience approach, Psychological Bulletin, 126, p. 109-137.

Maillat, D., \& S. Oswald (2009) Defining Manipulative Discourse: The Pragmatics of Cognitive Illusions, International Review of Pragmatics, 1, p. 348-370.

Micheli, R. (2010) L'émotion argumentée, Paris: Cerf.

Moeschler, J. (2009) Pragmatics, propositional and non-propositional effects. Can a theory of utterance interpretation account for emotions in verbal communication?, Social science information, 48, 3, p. 447-463.

Morency, P., Oswald S. \& L. de Saussure (2008) Explicitness, implicitness and commitment attribution: A cognitive pragmatic approach, Belgian journal of linguistics, 22, p. 197-220.

Petty, R. E. \& J. T. Cacioppo (1986) The elaboration likelihood model of persuasion, in Berkowitz L. (ed), Advances in Experimental Social Psychology, 19, p. 123-205.

Pinker, S., Nowak, M. A., \& J. J. Lee (2008) The logic of indirect speech, Proceedings of the National Academy of Sciences, 105, 3, p. 833-838.

Plantin, C. (2011) Les bonnes raisons des émotions, Berne: Peter Lang.

Potts, C. (2007) The expressive dimension, Theoretical Linguistics, 33, 2, p. 165-197.

Rey, G. (1980) Functionalism and the emotions, in Rorty, A. (ed) Explaining Emotions, Los Angeles: University of California Press, p. 163-198. 
de Saussure, L. (2005) Manipulation and Cognitive Pragmatics: Preliminary Hypotheses, in de Saussure, L. \& P. Schulz (eds), Manipulation and Ideologies in the Twentieth Century, Discourse, Language, Mind, Amsterdam-Philadelphia: John Benjamins, p. 113-146.

de Saussure, L. (2012) Compétence et contre-performance pragmatiques, Mémoires de la Société de Linguistique de Paris, 21, p. 135-159.

de Saussure, L. (2013) Perspectival interpretations of tenses, in Jaszczolt K. \& L. de Saussure (eds), Time: Language, cognition and reality, Oxford: Oxford University Press, p. 46-69.

de Saussure, L. (2018) The straw man fallacy as a prestige gaining device, in Oswald, S., Jacquin, J. \& T. Herman, Argumentation and Language, Amsterdam: Springer, p. 171-190.

de Saussure, L. \& S. Oswald (2009) Argumentation et engagement du locuteur. Pour un point de vue subjectiviste, Nouveaux cahiers de linguistique française, 29, p. 215-243.

de Saussure, L. \& P. Schulz (2009) Subjectivity out of irony, Semiotica, 173, 1, p. 397-416.

Sperber, H. (1914) Über den Affekt als Ursache der Sprachveründung, Halle a.d. Saale: Max Niemeyer. Sperber D. \& D. Wilson (1986) Relevance. Communication and cognition, Oxford: Basil Blackwell (2 ${ }^{\text {nd }}$ ed. 1995).

Sperber, D. \& D. Wilson (1998) The mapping between the mental and the public lexicon, in Carruthers, P. \& J. Boucher (eds) Thought and language, Cambridge: Cambridge University Press, p. 184-200.

Sperber, D. \& D. Wilson (2015) Beyond speaker's meaning, in Croatian Journal of Philosophy, XV, 44, p. $117-149$.

Sperber, D., Clément, F., Heintz, C., Mascaro, O., Mercier, H., Origgi, G. \& D. Wilson (2010) Epistemic vigilance, Mind and Language, 25, p. 359-393.

Tversky, A., \& D. Kahneman (1974) Judgment under Uncertainty: Heuristics and Biases, Science, 185, 4157, p. 1124-1131.

Wharton, T. (2003) Interjections, language and the 'showing'/'saying' continuum, Pragmatics and Cognition, 11-1, p. 39-91.

Wharton, T. (2009) Pragmatics and Non-Verbal Communication, Cambridge: Cambridge University Press.

Wharton, T. (2015) That bloody so-and-so has retired: expressives revisited, Lingua, 175-176, 2016, p. 20-35, DOI: 10.1016/j.lingua.2015.08.004.

Wharton, T. \& C. Strey (2017) Slave to the passions: making emotions relevant, in Carston, R., Clark, B. and K. Scott (eds) Relevance Pragmatics and Interpretation, Cambridge: Cambridge University Press.

Wilson, D. (1973) Presuppositions and non-truth-conditional semantics, Thèse de doctorat, MIT, ms, $241 \mathrm{p}$.

Wilson, D. (2015) Reassessing the conceptual-procedural distinction, Lingua, 175-176, 2016, p. 5-19, DOI:10.1016/j.lingua.2015.12.005. 


\section{NOTES}

1. Pour la sémantique formelle, rappelons que le sens des phrases est une (ou plusieurs) propositions, donc des pensées susceptibles d'une évaluation en vrai/faux. Dans cette tradition, la signification des phrases est donc l'ensemble des conditions qui les rendent vraies.

2. Frankfurter Allgemeine Zeitung, 11.11.1988, trad. Wikipedia. Cf. de Saussure (2012); Ensink (1997).

3. Notre position diffère donc des conceptions du sentiment que l'on trouve chez Damasio (2006/1994) et dans la théorie de l'évaluation cognitive (appraisal theory) dans ses diverses variantes (Ellsworth 2013; Frijda 2007; Lazarus 1991; Deonna and Teroni 2012).

4. cf. de Saussure (2005a); Maillat \& Oswald (2009) pour la notion de contrainte sur la construction du contexte.

5. Voir Wharton (2009) pour un argumentaire détaillé à ce sujet.

6. Voir les développements en sciences cognitives sur la gestion des états épistémiques chez Sperber \& al. (2010).

\section{RÉSUMÉS}

Dans cet article, nous proposons d'intégrer les effets émotionnels (à la suite de Wharton, 2003), à savoir l'expressivité, donc les aspects affectifs de la communication linguistique, dans une conception pragmatique cognitive générale, en l'occurrence la Théorie de la pertinence de Sperber \& Wilson (1986/95). Nous partons du constat que cette dimension cruciale de la communication linguistique a été pratiquement entièrement laissée de côté par le courant formel et propositionnaliste en sémantique et pragmatique. En nous appuyant sur quelques cas empiriques, et notamment sur celui d'un « malentendu » émotionnel, nous proposons de lier ces effets à la notion d'ineffabilité descriptive. Nous défendons l'idée que les effets émotionnels permettent de dépasser les limitations de la communication propositionnelle et interagit étroitement avec elle; nous proposons d'envisager ces effets comme atteignant leur pertinence par leur résonnance expérientielle, à savoir leur capacité d'évoquer des émotions similaires par l'accès à des traces mémorielles ou par l'imagination, ce qui ouvre à une forme particulière de créativité langagière.

Since the communication of information about emotional states clearly plays a central role in human interaction, it might be presumed that pragmatic accounts of linguistic communication would include well developed views on how these states are communicated. However, for a range of reasons, aspects of linguistic communication which feel as if they go beyond the strictly propositional dimension have long been dismissed by scholars interested in meaning: as a result, there is a conspicuous void in theories of pragmatics where the emotional dimension to communication should be. Although speech-act philosophers found ways to incorporate aspects of non-truth-conditional meaning in terms of propositional attitudes, the direct expression of emotional states, as opposed to the description of such states is largely ignored. Indeed, in most modern theories of pragmatics, the domain of research is limited to those cases that fall within the category of non-natural meaning. This excludes expressive meaning.

This paper synthesizes an account of emotions and emotion-reading that fits with work in a cognitive model of pragmatics - relevance theory (Sperber \& Wilson, 1986/1995) - and with work on emotions in cognitive and affective science (Cosmides \& Tooby, 2000; Deonna \& Teroni, 2012). 
Turning first to pragmatics, there are two main ways in which the relevance theory account of utterance interpretation diverges from both traditional Gricean and Neo-Gricean ones and we believe that these two divergences underpin our ultimate claim: that relevance theory is capable of rising to the challenges we raise in this chapter. The first of these is that within relevance theory the informative intention need not always be described as an intention to communicate a single proposition and propositional attitude. In relevance theory the informative intention is construed more broadly than merely an intention to communicate a proposition $p$ : as an intention 'to make manifest or more manifest to the audience a set of assumptions I' (Sperber and Wilson, 1986/1995: 58 - our italics). When what is communicated is quite vague, it typically involves a marginal increase in the manifestness of a very wide range of weakly manifest assumptions, resulting in an increased similarity between the cognitive environments of communicator and audience.

The second difference concerns the line Grice (1957) famously drew between showing and nonnatural meaning (meaning ${ }_{\mathrm{NN}}$ ). It has often been remarked that this line has had a huge influence on the development of pragmatics. Many pragmatists continue to focus on the notion of meaning $_{\mathrm{NN}}$ and abstract away from cases of showing. So where, in fact, should the line be drawn? According to relevance theory, it should not be drawn at all. Cases of both showing and meaning $_{\mathrm{NN}}$ qualify as instances of ostensive-inferential communication and instead of there being a cut-off between the two notions, there is a continuum of cases in between.

Turning to cognitive and affective sciences, we adopt two ideas. Firstly, from cognitive science, we view emotions as one type of evolved superordinate cognitive mechanism, the function of which is to mobilize cognitive processes responsible for perception and attention, physiological changes etc. Secondly, we endorse the key concept from affective science that emotions are attitudes bearing on evaluations (contrarily to raw feelings). So, rather than treating all stimuli as equal while scanning the environment, (which would, presumably, result in some kind of cognitive overload), appraisal theorists claim that people scan for inputs as a function of particular criteria, among which goal relevance. Attention is therefore paid to stimuli when once appraised as being relevant and, in certain conditions, an emotional episode may result.

Our account builds on these two observations using relevance-theoretic pragmatics. The kind of information conveyed during emotional communication puts the user into a state in which emotional procedures are highly activated, and are therefore much more likely to be recognised and selected by an audience (Wharton, 2009; de Saussure, 2013). Central to this thinking is the idea that the notion of cognitive effect needs to be complemented by a new notion of emotional effect, typically activated by emotion-reading procedures, which trigger immediate experiential responses either in memory or in imagination, which are straight away relevant - potentially very much so - in one's mind.

INDEX

Keywords : Relevance Theory, emotion, pragmatics, expressivity, non-propositional meaning

Mots-clés : théorie de la pertinence, émotion, pragmatique, expressivité, sens nonpropositionnel

\section{AUTEURS}

\section{LOUIS DE SAUSSURE}

Université de Neuchâtel (Suisse)

louis.desaussure@unine.ch 


\section{TIM WHARTON}

Université de Brighton (Royaume-Uni)

t.wharton@brighton.ac.uk 